\title{
BiGlobal and Point Vortex Methods for the Instability Analysis of Wakes
}

\author{
Juan A. Tendero*† and Pedro Paredes ${ }^{\dagger}$ \\ School of Aeronautics, Universidad Politécnica de Madrid, Madrid, 28040, Spain \\ Miquel Roura ${ }^{\ddagger}$ \\ Gamesa Innovation and Technology S.L., Madrid, 28043, Spain. \\ Rama Govindarajan ${ }^{\S}$ \\ TIFR Centre for Interdisciplinary Sciences, Hyderabad, 500075, India \\ Vassilios Theofilis ${ }^{\mathbb{1}}$ \\ School of Aeronautics, Universidad Politécnica de Madrid, Madrid, 28040, Spain
}

\begin{abstract}
To better understand destruction mechanisms of wake-vortices behind aircraft, the point vortex method for stability (inviscid) used by Crow is here compared with viscous modal global stability analysis of the linearized Navier-Stokes equations acting on a two-dimensional basic flow, i.e. BiGlobal stability analysis.

The fact that the BiGlobal method is viscous, and uses a finite area vortex model, gives rise to results somewhat different from the point vortex model. It adds more parameters to the problem, but is more realistic.
\end{abstract}

\section{Introduction}

$\mathrm{T}$ HE problem of aircraft wakes and how long they last before some mechanism destroys them has been widely studied. The importance of the problem arose a long time ago, with the appearance of the Boeing 747, and was again of importance when Airbus 380 came into service. However, in the present days, not only the hazard due to such big aircraft is important, also, as the air traffic increases, the air space becomes more and more saturated and it is of major importance to reduce the distances between aircraft to be able to increase the density of them in saturated air spaces.

Consequently, the acceleration of the destruction of wake vortices, although studied for a long time, is still an open problem. In the way to eliminate that hazard, the stability of the wake needs to be studied in depth as a previous stage, to be able to distinguish which configurations can last for longer and to understand the mechanism of its destruction.

An isolated vortex is known to last for very long, but, in general, aircraft wake destruction mechanisms use to involve various vortices, so this dissipation occurs faster. A very good example of that statement is the very well known Crow instability for a counter-rotating vortex pair. Crouch also found some other mechanisms of destruction that act faster for two vortex pairs as it will be the configuration of a plane with deployed flaps. Following this line, many other studies can be found that analyze vortex interaction for wake destruction. 
Here, two different methods are compared for the study of wake instabilities, the inviscid point vortex method for stability and the viscous modal stability analysis of the linearized Navier-Stokes equations using a basic flow in 2D (BiGlobal).

The first of them, which will be called inviscid, has two variants. One, applied to wakes by Kármán ${ }^{3,4}$ is a $2 \mathrm{D}$ analysis where the vortices are seen as points in a plane. The other, that involves many works, like Crow, ${ }^{1}$ Jiménez, ${ }^{5}$ Crouch $^{2}$ and Fabre et al. ${ }^{6}$ amongst others, is a $3 \mathrm{D}$ analysis where the vortices are seen as parallel filaments, initially straight and later with a sinusoidal perturbation.

The second method, much more modern in time, but maybe even more used, is viscous. There have been many successful ways to employ that method, but they encountered at least some difficulties, most of them involving the need of too much computational resources. Some works as Hein and Theofilis ${ }^{7}$ and Rodríguez and Theofilis ${ }^{8}$ have used Chebyshev-Gauss-Lobatto points, that provide high accuracy, but use a great amount of machine memory, specially when high number of grid points is needed, leading to the need of the use of big machine stations. This methods do not allow a great benefit when sparse algebra solvers are implemented, as the collocation matrices are dense and the complete matrices to solve do not have as much zeros as finite differences or other methods that do not imply full collocation matrices. Broadhurst et al. ${ }^{9}$ solved the problem using spectral/hp-element methods and González et al. ${ }^{10}$ used low order finite elements, which achieve good solutions but needed very complicated meshes. This time high order finite differences are used following the methodology of Paredes el al., ${ }^{11}$ which allows to obtain similar results with much less computational resources, because the use of high accuracy methods is possible for sparse matrices and then sparse algebra solvers can be implemented.

This work has been structured as follows: This first chapter is an introduction, followed by the second chapter that explains the theory behind both methods, the third chapter shows and comments the results obtained and finally some conclusions are presented in the last chapter.

\section{Theory}

The theory behind both inviscid vortex methods for stability analysis and viscous global modal analysis is presented in this chapter. The two methods expose some similarities, because both of them are based on the linearization of perturbed equations and the solution of an eigenvalue problem, but as the vortex methods are inviscid and based on the Biot-Savart equation, the standard analysis is a viscous method that is based on the Navier-Stokes equations. This difference in the equations leads to a difference in the variables, as these used in the vortex methods are the point or filament positions, while these of the viscous methods are the flow variables.

In addition to that, it should be noted that the Navier-Stokes are the equations that the flow follows, while the Biot-Savart relation applied to points or filaments with vorticity is only an idealization that will give good results only when the assumptions taken are valid. However, the solution of the Navier-Stokes, even linearized, gives more trouble, specially when $R e$ is high, in other words, when viscosity is less important. That is a reason to think that inviscid methods could be a good solution, and in fact are, as it has been proved along the history. Furthermore, inviscid methods use to require less computational resources and therefore, solutions are easier to obtain in most of the cases. However, it should be noted that this inviscid limit is not the same that the solution of the Navier-Stokes equations as $R e \rightarrow \infty$, because the highest derivative cannot go to zero. For that reason, one should never forget that inviscid methods solve an approximation which may have singularities in boundary layers and concentrate vortices, as well as turbulent mechanisms are neglected.

The analysis of this problem has followed different sets of nomenclature for the coordinate system. This paragraph exposes the options and the reasons to have chosen one in particular here. It is very common in the vortex field to use $y$ and $z$ as coordinates of the plane perpendicular to the vortex and $x$ as the direction of the vortex, as it is done by $\mathrm{Crow}^{1}$ and Crouch. ${ }^{2}$ That is usually done because the main direction is the direction of the vortex, but some other people, use other names for the three directions, as Jiménez that uses $e_{1}, e_{2}$ and $e_{3}$, being the third of them the direction of the vortex, or Rennich and Lele ${ }^{12}$ that uses $x$ and $y$ as the plane coordinates and $z$ for the vortex direction. The last of them will be used here, because it seems more general, when one applies the methods that will be explained below for more general cases. In addition, and maybe more important, a $2 \mathrm{D}$ view is extended and it is more clear when one uses the third coordinate to represent the perpendicular direction to this $2 \mathrm{D}$ point of view. 


\section{II.A. Point Vortex Linear Modal Analysis}

This method for stability assumes a given set of point vortices in a 2D plane that are in equilibrium. However, this assumption can be relaxed to a set of points that maintain their relative positions, as it occurs for the two counter-rotative vortices. Later a small perturbation is introduced and the equations of the movement linearized to first order in the perturbations. These linearized equations are not solved in time in this approach, instead, the temporal behavior is supposed to be in exponential form and, using this assumption, an eigenvalue problem is solved to analyze the stability of the problem.

This methodology, is similar to that used in standard global modal analysis, but here the equations of the movement are the equations that give the movement of the point vortices and the perturbed variables are the point positions, opposed to the Navier-Stokes equations and the flow variables that are used there.

The analysis mentioned is presented for $2 \mathrm{D}$ point vortices and perturbations, and later for the filaments in 3D that represent a set of points in 2D, but for sinusoidal perturbations in the third direction. This method in 3D was used by Crow to discover the wake instability that has now his name.

\section{II.A.1. Two Dimensional Perturbations Procedure, particularized for two counter-rotative vortices}

The starting point is a configuration of $N$ point vortices in a plane, $\mathbf{x}_{p}=\left[x_{p}, y_{p}\right]$, each of them denoted by the subindex $p$. This set of points will move following Eq. (1), where $\mathbf{e}_{\mathbf{z}}$ is the vector perpendicular to the plane.

$$
\mathbf{u}\left(\mathbf{x}_{p}\right)=-\frac{1}{2 \pi} \sum_{q=1, p \neq q}^{N} \Gamma_{q} \frac{\left(\mathbf{x}_{p}-\mathbf{x}_{q}\right) \times \mathbf{e}_{\mathbf{z}}}{\left|\mathbf{x}_{p}-\mathbf{x}_{q}\right|^{2}}
$$

Perturbations of the form of $\mathbf{x}_{p}=\mathbf{X}_{p}+\varepsilon \mathbf{x}_{p}^{\prime}$ are introduced in the vortex positions, and later the resulting equations are linearized, leaving only terms of order $\varepsilon$. In order to do that, $\mathbf{u}_{p}=\left[u_{p}, v_{p}\right]=$ $\mathrm{d} \mathbf{x}_{p} / \mathrm{d} t=\mathbf{u}\left(\mathbf{x}_{p}\right)-\overline{\mathbf{U}}$, where $\overline{\mathbf{U}}$ is in this case the descent speed of the pair. Note that $\mathbf{X}_{p}$ represent an equilibrium configuration, and therefore, the order one part of the equation is satisfied and gives the value of $\overline{\mathbf{U}}=-\left(\boldsymbol{\Gamma}_{a} / 4 \pi X_{a}\right) \mathbf{e}_{\mathbf{z}}$ and the order $\varepsilon$ part of the equation remain unchanged. Time derivatives are supposed homogeneous as $\mathbf{x}_{p}^{\prime}=\hat{\mathbf{x}}_{p} e^{-i \omega t} . N=2$ is substituted for the case of two counter rotative vortices. Here, $2 X_{a}$ is the distance between the vortices and $\Gamma_{a}$ the circulation of the right vortex, being the opposite for the left.

$$
\begin{array}{r}
-\mathrm{i} \omega \hat{x}_{1}=\frac{\Gamma_{a}}{2 \pi}\left(\frac{\hat{y}_{1}-\hat{y}_{2}}{\left(2 X_{a}\right)^{2}}\right) \\
-\mathrm{i} \omega \hat{y}_{1}=\frac{\Gamma_{a}}{2 \pi}\left(\frac{\hat{x}_{1}-\hat{x}_{2}}{\left(2 X_{a}\right)^{2}}\right) \\
-\mathrm{i} \omega \hat{x}_{2}=-\frac{\Gamma_{a}}{2 \pi}\left(\frac{\hat{y}_{2}-\hat{y}_{1}}{\left(2 X_{a}\right)^{2}}\right) \\
-\mathrm{i} \omega \hat{y}_{2}=-\frac{\Gamma_{a}}{2 \pi}\left(\frac{\hat{x}_{2}-\hat{x}_{1}}{\left(2 X_{a}\right)^{2}}\right)
\end{array}
$$

It is time to non-dimensionalize these equations. The distance between the vortices will be the length for this purpose, as well as the circulation of the right vortex will be the circulation used for the same. Using this non-dimensionalization, time will be non-dimensionalized by $2 \pi\left(2 X_{a}\right)^{2} / \Gamma_{a}$. Note that this time is the time needed to propagate a distance $2 X_{a}$ and is not the same non-dimensionalization that is usually used for two co-rotative vortices that is usually that, multiplied by $\pi$, that is the time for the vortices to make one turn.

$$
\begin{aligned}
& \tilde{\omega}=\frac{2 \pi \omega\left(2 X_{a}\right)^{2}}{\Gamma_{a}} \\
& \tilde{x}_{i}=\frac{\hat{x}_{i}}{2 X_{a}} \\
& \tilde{y}_{i}=\frac{\hat{y}_{i}}{2 X_{a}}
\end{aligned}
$$


Using this non-dimensionalization, the eigenvalue problem has the following form:

$$
-\mathrm{i} \tilde{\omega}\left(\begin{array}{c}
\tilde{x}_{1} \\
\tilde{y}_{1} \\
\tilde{x}_{2} \\
\tilde{y}_{2}
\end{array}\right)=\left(\begin{array}{cccc}
0 & 1 & 0 & -1 \\
1 & 0 & -1 & 0 \\
0 & 1 & 0 & -1 \\
1 & 0 & -1 & 0
\end{array}\right)\left(\begin{array}{c}
\tilde{x}_{1} \\
\tilde{y}_{1} \\
\tilde{x}_{2} \\
\tilde{y}_{2}
\end{array}\right)
$$

\section{II.A.2. Three Dimensional Perturbations Procedure, particularized for two counter-rotative vortices}

The starting point is again a configuration of $N$ point vortices in a plane, but now, they are seen in a three dimensional point of view. These points are actually infinite lines of concentrated vorticity perpendicular to that plane. The set of filaments will move following Eq. (7), where similarities can be seen with Eq. (1), showing that the only differences are the change of the direction of the filaments, that now is more generic than only $\mathbf{e}_{\mathbf{z}}$, shown as $\mathrm{dl}_{q}$, and the integration along the filament, which involves some slight changes. The most important factor to have into account is the fact that one filament can interact with itself and therefore, the constraint $p \neq q$ has been removed. However, one point of the filament cannot interact with itself, because the integrals will diverge if that happens, and accordingly, this possibility has to be accounted in the integral. There are various ways to overcome that difficulty, the most common is what is called the cutoff integral, which consist in eliminating a small portion from the integral. The length of the portion that is eliminated or cut, is what is called the cutoff parameter, which is a length that has to be of the order of the size of the core of the vortex. Consequently, the integral has to be calculated as a cutoff integral, or, otherwise, it would diverge when it is calculated along a filament over itself. Several approaches are made in literature for the value of this cutoff, as well as other approximations, see Crow- and Widnall et al.--

$$
\mathbf{u}\left(\mathbf{x}_{p}\right)=-\frac{1}{4 \pi} \sum_{q=1}^{N} \Gamma_{q} \int_{l} \frac{\left(\mathbf{x}_{p}-\mathbf{x}_{q}\right) \times \mathrm{d} \mathbf{l}_{q}}{\left|\mathbf{x}_{p}-\mathbf{x}_{q}\right|^{3}}
$$

Here, again, as it was done in the 2D problem, the vortex positions are perturbed and the equations are linearized. What is said there is valid here, but, although the perturbations and the equilibrium positions are 2D again, it should be noted that the problem is regarded as 3D and the perturbations depend upon the third direction. This dependence is assumed homogeneous and the perturbations have the shape $\mathbf{x}_{p}^{\prime}=\hat{\mathbf{x}}_{p} \mathrm{e}^{\mathrm{i} k z_{p}-\mathrm{i} \omega t}$. However, this time, the process is not straightforward. $\mathrm{dl}_{q}=\left(\mathbf{e}_{\mathbf{z}}+\varepsilon \partial \mathbf{x}_{q}^{\prime} / \partial z_{q}\right) \mathrm{d} z_{q}$, and new combinations of terms of order $\varepsilon$ appear. Furthermore, as it was mentioned, the integrals that have to be calculated diverge and they have to be seen as cutoff integrals. In addition to that, the substitution of $\mathbf{u}\left(\mathbf{x}_{p}\right)$ introduces more complications. This time, $\mathbf{u}_{p}=\mathbf{u}\left(\mathbf{x}_{p}\right)-\overline{\mathbf{U}}=\left[u_{p}, v_{p}, w_{p}\right]$, and the derivatives come from the equation $\mathrm{d} \mathbf{x}_{p} / \mathrm{d} t+w_{p} \mathrm{~d} \mathbf{x}_{p} / \mathrm{d} x=\mathbf{e}_{\mathbf{x}} u_{p}+\mathbf{e}_{\mathbf{y}} v_{p}+\mathbf{e}_{\mathbf{z}} w_{p}$, where it is observed that $z$ is the only directional dependence. When this equation is linearized, the convection term is of higher order, and therefore it is the $\mathrm{d} z_{p}^{\prime} / \mathrm{d} t$ term that can be neglected. In order to calculate the solution for two counter rotating vortices, again $2 X_{a}$ is assumed to be the distance between the vortices and $\Gamma_{a}$ the circulation of the right vortex. $d$ is the value of the cutoff parameter, assumed to be equal for the two vortices, which should be of the order of the real vortex core. The solutions of the integrals are represented using the modified Bessel functions of the second kind $K_{0}(\alpha)$ and $K_{1}(\alpha)$, and the integral cosine, $\mathrm{Ci}(\alpha)$.

$$
\begin{aligned}
-\mathrm{i} \omega \hat{x}_{1}=\frac{\Gamma_{a}}{2 \pi} & {\left[\left(\frac{1}{\left(2 X_{a}\right)^{2}}+\frac{\cos k d-1+k d \sin k d-k^{2} d^{2} C i(k d)}{2 d^{2}}\right) \hat{y}_{1}\right.} \\
& \left.-\left(k^{2} K_{0}\left(2 k X_{a}\right)+\frac{k K_{1}\left(2 k X_{a}\right)}{2 X_{a}}\right) \hat{y}_{2}\right] \\
-\mathrm{i} \omega \hat{y}_{1}=\frac{\Gamma_{a}}{2 \pi} & {\left[\left(\frac{1}{\left(2 X_{a}\right)^{2}}-\frac{\cos k d-1+k d \sin k d-k^{2} d^{2} C i(k d)}{2 d^{2}}\right) \hat{x}_{1}-\frac{k K_{1}\left(2 k X_{a}\right)}{2 X_{a}} \hat{x}_{2}\right] } \\
-\mathrm{i} \omega \hat{x}_{2}=\frac{\Gamma_{a}}{2 \pi} & {\left[\left(-\frac{1}{\left(2 X_{a}\right)^{2}}-\frac{\cos k d-1+k d \sin k d-k^{2} d^{2} C i(k d)}{2 d^{2}}\right) \hat{y}_{2}\right.} \\
& \left.+\left(k^{2} K_{0}\left(2 k X_{a}\right)+\frac{k K_{1}\left(2 k X_{a}\right)}{2 X_{a}}\right) \hat{y}_{1}\right] \\
-\mathrm{i} \omega \hat{y}_{2}=\frac{\Gamma_{a}}{2 \pi} & {\left[\left(-\frac{1}{\left(2 X_{a}\right)^{2}}+\frac{\cos k d-1+k d \sin k d-k^{2} d^{2} C i(k d)}{2 d^{2}}\right) \hat{x}_{2}+\frac{k K_{1}\left(2 k X_{a}\right)}{2 X_{a}} \hat{x}_{1}\right] }
\end{aligned}
$$


The equations can be non-dimensionalized using again the same time $\left(2 \pi\left(2 X_{a}\right)^{2} / \Gamma_{a}\right)$ and distance $\left(2 X_{a}\right)$, where the same comments apply one more time. However, in this case, other two parameters will appear, because there are two more lengths in the problem, $d$ and $1 / k$ :

$$
\begin{aligned}
& \tilde{d}=\frac{d}{2 X_{a}} \\
& \tilde{k}=2 X_{a} k
\end{aligned}
$$

Using this non-dimensionalization, the eigenvalue problem obtained is the same as that obtained in Eqs. (8) of Crow, but the notation has changed, the coordinates $[x, y, z]$, used here, are interchanged by $[y, z, x]$ there and also vortex numbers 1 and 2 are interchanged. In a similar fashion as in that paper, a function $\tau(\theta)=\cos \theta-1+\theta \sin \theta-\theta^{2} \operatorname{Ci}(\theta)$ is defined to shorten the equations shown.

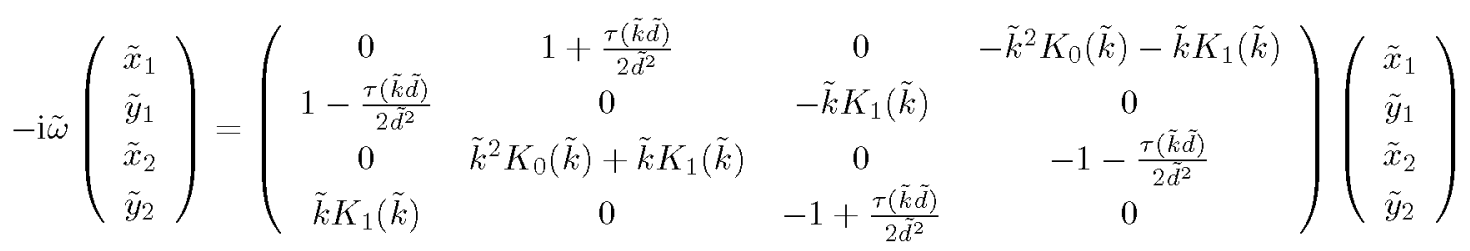

\section{II.B. Modal Linear Stability Theory}

Modal linear stability studies the asymptotic behavior of the flow to small disturbances. The linearized problem is converted into an eigenvalue problem and stability is given by the sign of the real part of the eigenvalues. The process to derive the equations used here can be found in many works as Theofilis, ${ }^{14,15}$ although more details of the equations are given this time.

Three-dimensional Navier-Stokes equations of a viscous, incompressible fluid in dimensionless form and Cartesian coordinates are

$$
\nabla \cdot \overline{\mathbf{u}}=0, \quad \frac{\partial \overline{\mathbf{u}}}{\partial t}+\overline{\mathbf{u}} \cdot \nabla \overline{\mathbf{u}}=-\nabla \bar{p}+\frac{1}{R e} \nabla^{2} \overline{\mathbf{u}}
$$

with the Reynolds number defined as:

$$
R e=U_{L} L / \nu,
$$

being $U_{L}$ and $L$ the velocity and length scale respectively, used to non-dimensionalize the problem, and $\nu$ the kinematic viscosity.

The vector of fluid variables $\overline{\mathbf{q}}=[\overline{\mathbf{u}}, \bar{p}]^{T}$ is decomposed into a steady mean flow $\mathbf{Q}$ and an unsteady small disturbance or perturbation $\varepsilon \mathbf{q}$ :

$$
\overline{\mathbf{q}}(\mathbf{x}, t)=\mathbf{Q}(\mathbf{x})+\varepsilon \mathbf{q}(\mathbf{x}, t) .
$$

where $\varepsilon \ll 1$.

Introducing this decomposition of the perturbed flow into the Eq. (12), and subtracting the basic flow (as it satisfies the Navier-Stokes and continuity equations itself) one arrives to the so-called perturbation equations or Linearized Navier Stokes Equations (LNSE)

$$
\nabla \cdot \mathbf{u}=0, \quad \frac{\partial \mathbf{u}}{\partial t}+\mathbf{U} \cdot \nabla \mathbf{u}+\mathbf{u} \cdot \nabla \mathbf{U}=-\nabla p+\frac{1}{R e} \nabla^{2} \mathbf{u} .
$$

In these equations the $\mathcal{O}(\varepsilon)$ terms have been retained, while the non-linear perturbation term $(\mathbf{u} \nabla \mathbf{u})$ is $\mathcal{O}\left(\varepsilon^{2}\right)$ and can be neglected. The linearization is correct while the disturbance amplitude remains small enough for the non-linear terms to be negligible. This statement should be kept in mind as the true non-linear system can become unstable under finite-amplitude disturbances, under conditions in which the linearized system will stay stable. One physical example of this is the laminar-turbulent transition originated by the transient growth of initially small disturbances, that can be stable in a linear approach (see Butler for a review). 
In Cartesian coordinates, Eqs. (15) have the form of Eqs. (16). Where $\mathbf{u}=[u, v, w], \mathbf{U}=[U, V, W]$ and sub-index indicate derivatives.

$$
\begin{aligned}
u_{t}+U u_{x}+V u_{y}+W u_{z}+u U_{x}+v U_{y}+w U_{z} & =-p_{x}+\frac{1}{R e}\left(u_{x x}+u_{y y}+u_{z z}\right) \\
v_{t}+U v_{x}+V v_{y}+W v_{z}+u V_{x}+v V_{y}+w V_{z} & =-p_{y}+\frac{1}{R e}\left(v_{x x}+v_{y y}+v_{z z}\right) \\
w_{t}+U w_{x}+V w_{y}+W w_{z}+u W_{x}+v W_{y}+w W_{z} & =-p_{z}+\frac{1}{R e}\left(w_{x x}+w_{y y}+w_{z z}\right) \\
u_{x}+v_{y}+w_{z} & =0
\end{aligned}
$$

In linear stability theory, the perturbation term is usually written as the product of an amplitude function and a phase function, $\mathbf{q}=\hat{\mathbf{q}} \Theta$. Table (1) summarizes the different instability approaches arranged by increasing constrains to the basic flow. These different approaches are explained in the next subsections in more detail.

Table 1. Classification of linear stability theory concepts for analysis of a steady state $Q$.

\begin{tabular}{lclll} 
Denomination & Assumptions & Basic State & Amplitude & Phase Function $\Theta$ \\
\hline TriGlobal & - & $\mathbf{Q}\left(x_{1}, x_{2}, x_{3}\right)$ & $\hat{\mathbf{q}}\left(x_{1}, x_{2}, x_{3}\right)$ & $\mathrm{e}^{-\mathrm{i} \omega t}$ \\
BiGlobal & $\partial_{3} \mathbf{Q}=0$ & $\mathbf{Q}\left(x_{1}, x_{2}\right)$ & $\hat{\mathbf{q}}\left(x_{1}, x_{2}\right)$ & $\mathrm{e}^{\mathrm{i}\left(k_{3} x_{3}-\omega t\right)}$ \\
OSE & $\partial_{2} \mathbf{Q}=\partial_{3} \mathbf{Q}=0$ & $\mathbf{Q}\left(x_{1}\right)$ & $\hat{\mathbf{q}}\left(x_{1}\right)$ & $\mathrm{e}^{\mathrm{i}\left(k_{2} x_{2}+k_{3} x_{3}-\omega t\right)}$
\end{tabular}

Using that approach, what is finally obtained is an eigenvalue problem for $\omega$ as eigenvalues and $\hat{\mathbf{q}}$ as eigenvectors. The eigenvalues will have a real part, that will be the growth rate and a imaginary part that will be the frequency of the oscillations. In the notation used, $\omega$ is multiplied by $-i$, that means that the real part of $\omega$ will actually be the frequency changed of sign, and the growth rate will be the imaginary part. This option here, is called temporal amplification theory, and it is the only one applicable to TriGlobal. For the other two approaches, however, there are also wavenumbers $\left(k_{i}\right)$ that have to be taken into account. For temporal theory, they have to be all given and real, in order to have sinusoidal movement in the homogeneous directions. This method gives the growth or decay in time of the perturbations.

On the other hand, it exists the spatial theory. It consist of analyzing the problem using one of the wavenumbers as complex eigenvalues and leaving the frequency real. When spatial theory is selected, growing or decay may be found in the direction of the wavenumber that is calculated, given a frequency of oscillation. It cannot be used in TriGlobal, because it does not have any homogeneous direction. If the OSE approach is taken, it needs to be given a real wavenumber for one of the homogeneous directions. Furthermore, it should be taken into account that directional derivatives are second order, and then, the eigenvalue problem to solve will have eigenvalues to the power of two, with the consequent difficulties of solution. This complication, however, does not add significant aspects to the matter and will not be discussed any further in this work. For an interesting review of temporal and spatial theory, applied to local stability, see Mack. ${ }^{17}$

\section{II.B.1. Two Dimensions, BiGlobal}

If the dependence in the third direction is neglected, the two-dimensional parallel flow is assumed and the corresponding stability analysis is called BiGlobal instability (see Theofilis ${ }^{14,15}$ for a review). Assuming that the basic flow is now dependent on two out of the three spatial coordinates (see table 1):

$$
\mathbf{Q}=[U, V, W, P]^{T}(x, y),
$$

the coefficients of the LNSE. (Eq. (15)) are $z$-independent, and modal perturbations now get the form

$$
\mathbf{q}(x, y, z, t)=\hat{\mathbf{q}}(x, y) \mathrm{e}^{\mathrm{i}(\beta z-\omega t)}
$$

All this, is substituted into Eqs. (15), to obtain Eqs. (19). Again, some changes of notation are applied in that equation, $x_{1}, x_{2}$ and $x_{3}$ are called $x, y$ and $z$, while $k_{3}$ is called $\beta$. Derivatives are shown as sub-index, 
and now there are derivatives in two dimensions.

$$
\begin{aligned}
-i \omega \hat{u}+U \hat{u}_{x}+V \hat{u}_{y}+\mathrm{i} \beta W \hat{u}+\hat{u} U_{x}+\hat{v} U_{y} & =-\hat{p}_{x}+\frac{1}{R e}\left(\hat{u}_{x x}+\hat{u}_{y y}-\beta^{2} \hat{u}\right) \\
-i \omega \hat{v}+U \hat{v}_{x}+V \hat{v}_{y}+\mathrm{i} \beta W \hat{v}+\hat{u} V_{x}+\hat{v} V_{y} & =-\hat{p}_{y}+\frac{1}{R e}\left(\hat{v}_{x x}+\hat{v}_{y y}-\beta^{2} \hat{v}\right) \\
-i \omega \hat{w}+U \hat{w}_{x}+V \hat{w}_{y}+\mathrm{i} \beta W \hat{w}+\hat{u} W_{x}+\hat{v} W_{y} & =-\mathrm{i} \beta \hat{p}+\frac{1}{R e}\left(\hat{w}_{x x}+\hat{w}_{y y}-\beta^{2} \hat{w}\right) \\
\hat{u}_{x}+\hat{v}_{y}+\mathrm{i} \beta \hat{w} & =0
\end{aligned}
$$

This can be written as an eigenvalue problem with $[\hat{u}, \hat{v}, \hat{w}, \hat{p}]^{T}$ as eigenvectors and $\omega$ as eigenvalues. The disturbances are three-dimensional, but a sinusoidal dependence is assumed only in the homogeneous $z$-direction, with the periodicity length $L_{z}=2 \pi / \beta$. Eqs. (19) can be again written in matrix form.

$$
\left(\begin{array}{cccc}
\mathcal{L}_{2 D}+U_{x} & U_{y} & 0 & \mathcal{D}_{x} \\
V_{x} & \mathcal{L}_{2 D}+V_{y} & 0 & \mathcal{D}_{y} \\
W_{x} & W_{y} & \mathcal{L}_{2 D} & \mathrm{i} \beta \\
\mathcal{D}_{x} & \mathcal{D}_{y} & \mathrm{i} \beta & 0
\end{array}\right)\left(\begin{array}{c}
\hat{u} \\
\hat{v} \\
\hat{w} \\
\hat{p}
\end{array}\right)=\left(\begin{array}{cccc}
\mathrm{i} \omega & 0 & 0 & 0 \\
0 & \mathrm{i} \omega & 0 & 0 \\
0 & 0 & \mathrm{i} \omega & 0 \\
0 & 0 & 0 & 0
\end{array}\right)\left(\begin{array}{c}
\hat{u} \\
\hat{v} \\
\hat{w} \\
\hat{p}
\end{array}\right)
$$

where $\mathcal{L}_{2 D}=U \mathcal{D}_{x}+V \mathcal{D}_{y}+W \mathrm{i} \beta-\frac{1}{R e}\left(\mathcal{D}_{x x}+\mathcal{D}_{y y}-\beta^{2}\right)$. Here $\beta$ is a wavenumber parameter, related with the periodicity length along the homogeneous spatial direction, $z$.

\section{Results}

The results are collected in this section for the two vortex wake. First a simple 2D point vortex analysis is done, later a 3D filament vortex analysis is carried out, which is the same as Crow, and later the same problem is studied by means of standard linear modal stability (BiGlobal).

\section{III.A. 2D Point Vortex Analysis of Two Counter Rotative Vortices}

If the eigenvalues of the problem shown in Eq. (6) are calculated analytically, it is obtained that there is only one quadruple eigenvalue $-\mathrm{i} \tilde{\omega}=0$. Note that a numerical algorithm may fail here due to the singularity of the matrix. These four null eigenvalues means that if any perturbation is introduced, it will be maintained, neither amplified or damped, and, for any perturbation of the initial configuration the distance perturbed will remain constant. Due to the simplicity of the problem, this conclusion could have been obtained by simple deduction, without any mathematical analysis, but it is always important to find the mathematical proof of the results.

\section{III.B. 3D Point Vortex Analysis of Two Counter Rotative Vortices}

In this case, the eigenvalue problem that defines the problem to analyze, is given by Eq. (11). However, this equation depends of two parameters, $\tilde{k}$ and $\tilde{d}$, so they have been defined before. Once values are given to the two of them, the solution is straightforward. However, the physic of the problem has to be in mind for the selection of the parameters, as any random selection may not be physical. This section solves, basically, the same problem as Crow, but more details of the solution are obtained, which will be of great use to compare with other methods.

The cutoff distance will be related with the vortex core. The actual value for this relation is not easy to obtain, but several approaches have been made. In his paper, Crow gives a value of the ratio cutoff by vortex radious of around one third and, as the vortex cores are not supposed to interact with each other, the distance of the vortices has to be bigger (or much bigger) that the double of the vortex radious. This statement can be summarized saying that $\tilde{d}$ should be smaller than around 0.2 . This condition may be relaxed if the ratio between the cutoff and the vortex core is increased, but in any case, values of $\tilde{d}$ that approach to one are not realistic. This limit is imposed because the vortex cores should not touch each other.

Now, other restriction should be addressed. The oscillation of the vortices cannot be so small that the length of oscillation is smaller than the vortex core, because in this case, the oscillation would be inside the cutoff distance that has been extracted from the problem. The previous statement could be written as 
$1 / \tilde{k}>\tilde{d}$, which means that for a given $\tilde{d}, \tilde{k}$ should be approximately smaller than the inverse of it. For example, for a value of the cutoff of 0.1 , the wavenumber should be less than 10 and for a cutoff of 0.5 , the wavenumber should be less than 2 .

Note that the limits obtained above are approximated values and they could be exceeded, but they should be always keep in mind.

The eigenvalue problem is now solved for $\tilde{k}$ from 0 to 6 and five selections of $\tilde{d}$ : $0.1,0.2,0.3,0.4$ and 0.5 . Note that $\tilde{d}=0.2$ is in the limit of validity of the parameter and the higher values will be outside this limit. However, the results of these parameters are obtained to see how the problem changes when the cutoff does, but it should not be forgotten that the validity of the results in that range is at least doubtful. The resulting values of the amplification rate $\left(\tilde{\omega}_{i}\right)$ and frequency $\left(\tilde{\omega}_{r}\right)$ have been collected in figures 1 and 2 , where the upper half of figures $1(\mathrm{c}), 1(\mathrm{e})$ and $2(\mathrm{a})$ coincide with figure 9 of Crow.

The four eigenvalues are always in pairs and either they are real or imaginary, but they have never the two components. The couples have the same value with positive and negative sign. That is, when there is a growing mode, there exist a decaying mode, and the neutral eigenvalues appear as a complex plus a complex conjugate.

Lets introduce now the shape of the modes with the help of figures 3,4 and 5 . The first mode that grows for small $\tilde{k}$ is always a symmetric mode, shown in figures 4(a) and $5(\mathrm{a})$. For small $\tilde{d}$ it appears far from the asymmetric growing mode, but as $\tilde{d}$ increases, this mode, represented in figure $5(\mathrm{c})$, moves closer to small wavenumbers, appearing from any positive $\tilde{k}$ for a cutoff equal to 0.5 as figure 2(c) shows. Also for small cutoff parameters, a second region of symmetric instability appears near to the region of asymmetric instability, but this region approaches faster to the first region of instability as the cutoff increases becoming a single symmetric instability region for a value in between 0.2 and 0.3 of the cutoff distance. In the regions that either the symmetric or asymmetric mode are neutrally stable, two modes with frequency appear, that neither grow or decay, but move at a definite frequency in time both upstream and downstream.

To conclude this section of results, it could be said that the results as the wavenumber tends to zero are also zero, as was proved in the $2 \mathrm{D}$ analysis carried out previously.

\section{III.C. BiGlobal Analysis of Two Counter Rotative Vortices}

The BiGlobal analysis consists basically of solving the eigenvalue problem defined in Eq. (20). However, this solution is not straightforward and some considerations have to be done before. The way to discretize the derivatives is important and various approaches are possible. Chebyshev-Gauss-Lobatto discretization is very common, but has some drawbacks as the matrices that uses are full and then is less suitable for sparse algebra. On the contrary, high order finite differences can be used with high accuracy (see Paredes et al. ${ }^{11}$ ) and they can take all the advantage of sparse solvers. This discretization method is an essential tool to be able to obtain quality results in standard computers. The way to calculate the two dimensional operators is done by the Kronecker product, but that and other considerations related to the high order finite differences and its discretization are general of the method and can be seen in more detail in the mentioned paper by Paredes et al. ${ }^{11}$ In addition, some boundary conditions have to be imposed to the problem, which is a delicate aspect that will be analyzed in depth. In addition, a basic flow and a wavenumber $\beta$ have to be selected, aspect, the first of them, that will be commented later. It is also important to mention that an Arnoldi algorithm is used to obtain only a few eigenvalues in much shorter time, decreasing also the problem size.

\section{III.C.1. Basic Flow}

The basic flow is a field for $U, V, W$ and its derivatives, which must be a stationary solution of the NavierStokes equations. Sometimes it could be given analytically, and it could also be obtained as a solution of a 2D DNS code when the final result do not vary in time.

In order to study the stability of the two counter rotative vortices, two Lamb-Oseen vortices, Eqs. (21), can be introduced if a model without axial flow wants to be studied. If a model with axial flow is preferred, a $q$-vortex model, Eqs. (22), for the vortices can be supposed. These equations are usually written using the 


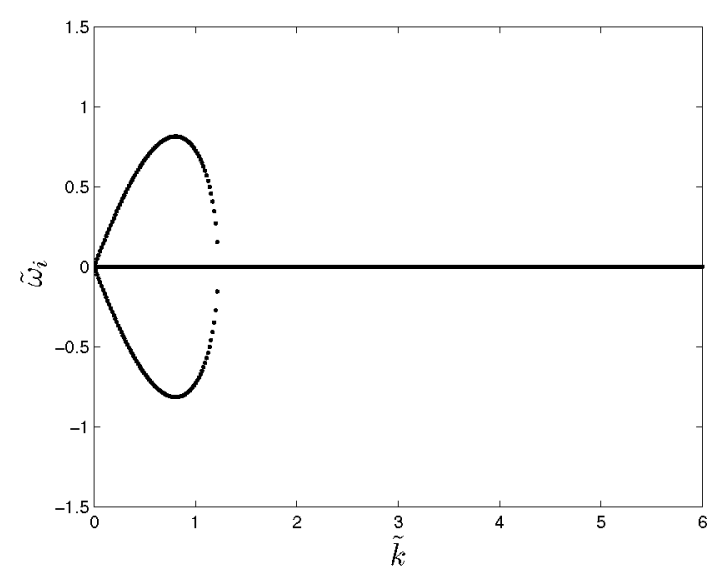

(a) Amplification rate $\left(\tilde{\omega}_{i}\right), \tilde{d}=0.1$.

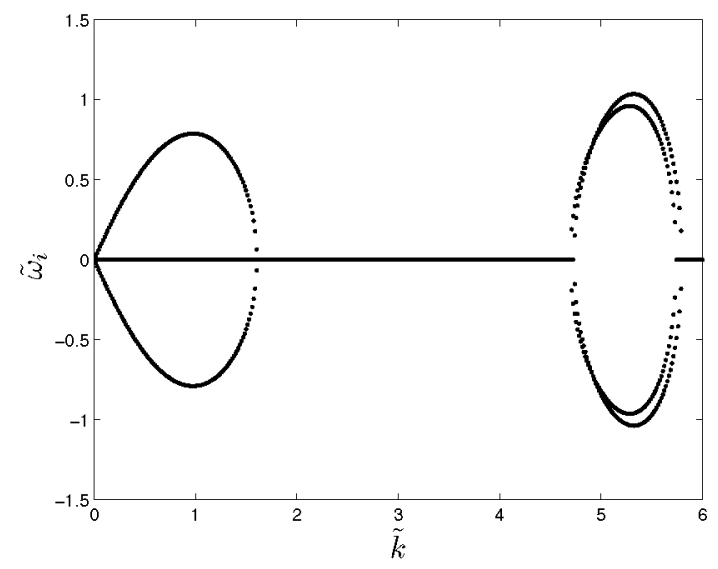

(c) Amplification rate $\left(\tilde{\omega}_{i}\right), \tilde{d}=0.2$.

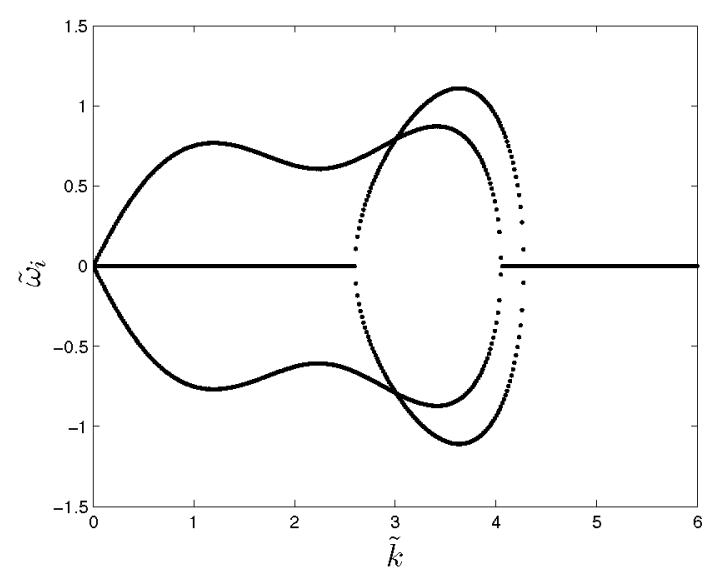

(e) Amplification rate $\left(\tilde{\omega}_{i}\right), \tilde{d}=0.3$.

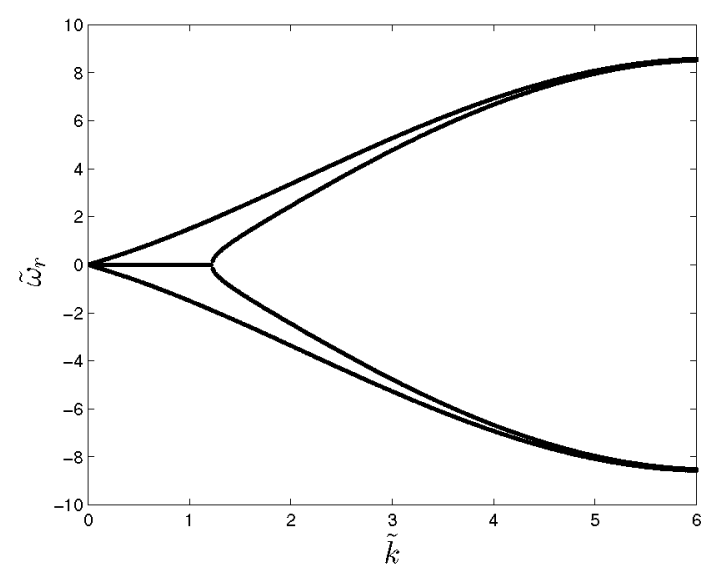

(b) Frequency $\left(\tilde{\omega}_{r}\right), \tilde{d}=0.1$.

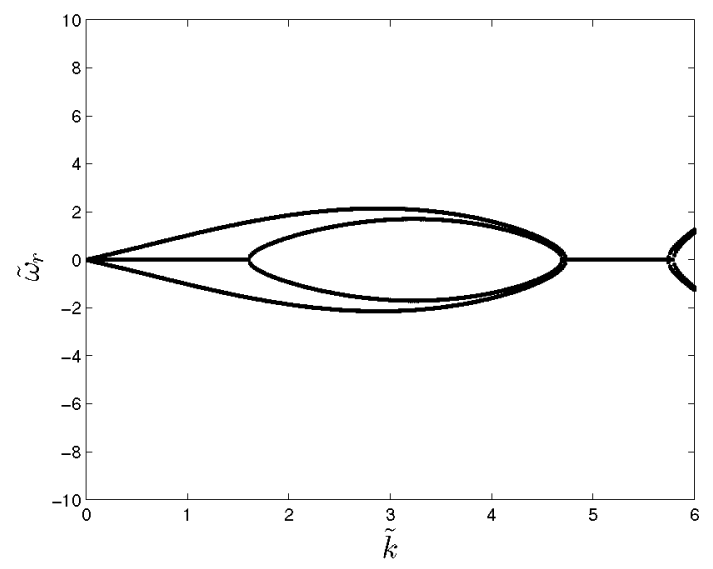

(d) Frequency $\left(\tilde{\omega}_{r}\right), \tilde{d}=0.2$.

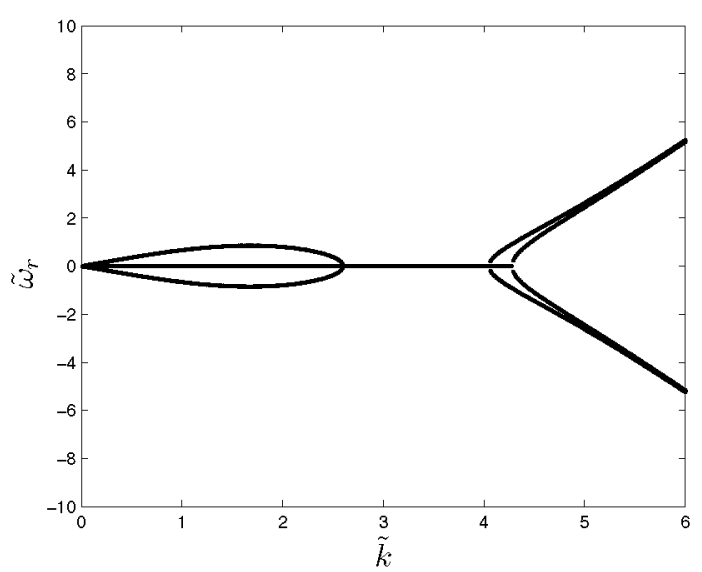

(f) Frequency $\left(\tilde{\omega}_{r}\right), \tilde{d}=0.3$.

Figure 1. Amplification rate $\left(\tilde{\omega}_{i}\right)$ and frequency $\left(\tilde{\omega}_{r}\right.$ as a function of the wavenumber $\tilde{k}$ for $\tilde{d}: 0.1,0.2$ and 0.3 . 


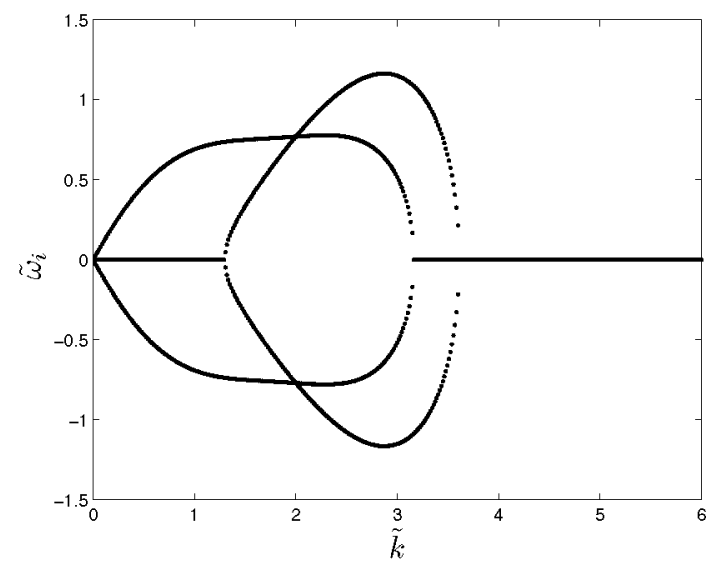

(a) Amplification rate $\left(\tilde{\omega}_{i}\right), \tilde{d}=0.4$.

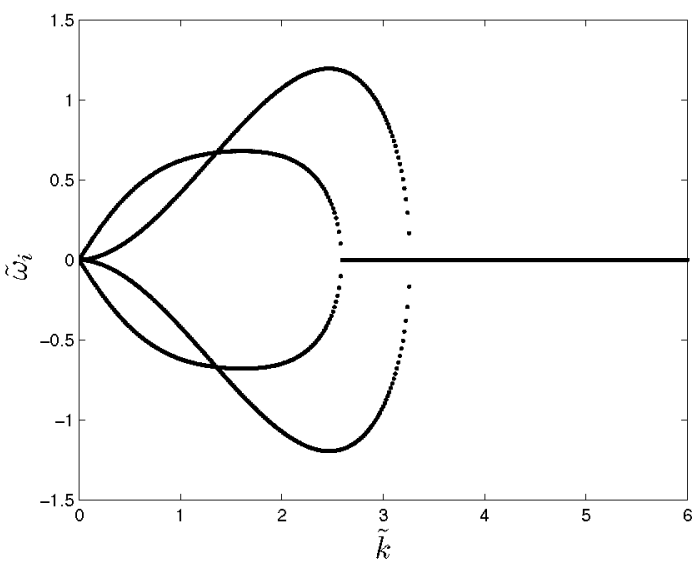

(c) Amplification rate $\left(\tilde{\omega}_{i}\right), \tilde{d}=0.5$.

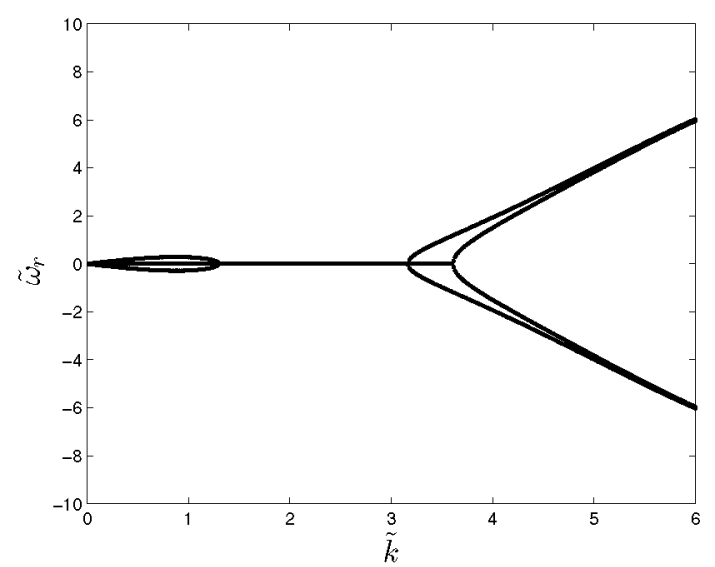

(b) Frequency $\left(\bar{\omega}_{r}\right), \tilde{d}=0.4$.

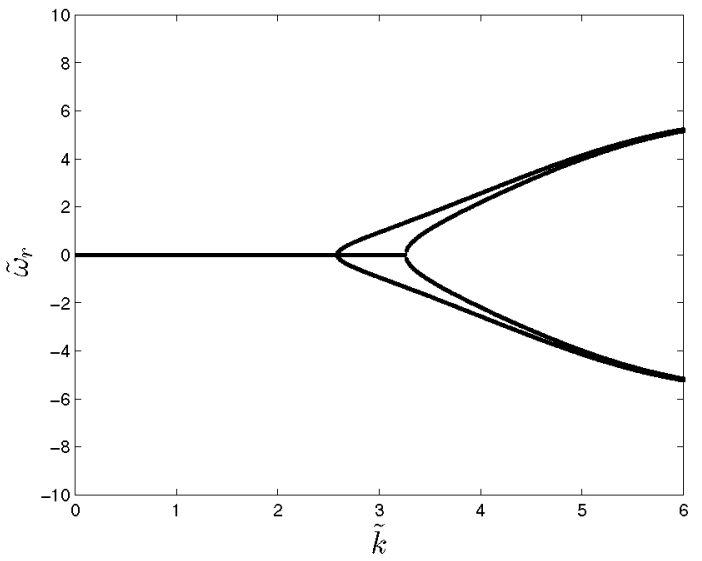

(d) Frequency $\left(\tilde{\omega}_{r}\right), \tilde{d}=0.5$

Figure 2. Amplification rate $\left(\tilde{\omega}_{i}\right)$ and frequency $\left(\tilde{\omega}_{r}\right.$ as a function of the wavenumber $\tilde{k}$ for $\tilde{d}: 0.4$ and 0.5 .

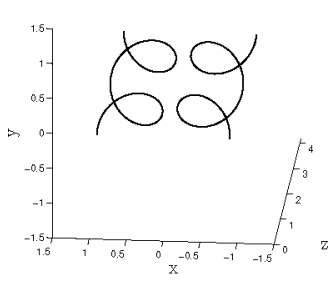

(a) Symmetric, positive

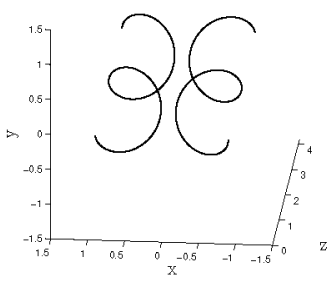

(b) Symmetric, negative

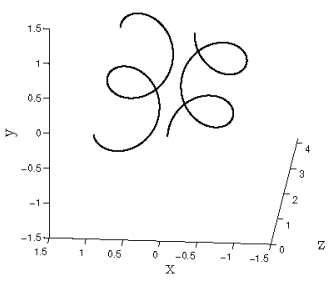

(c) Asymmetric, positive

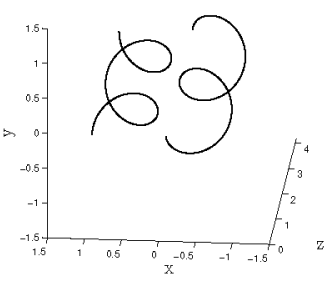

(d) Asymmetric, negative

Figure 3. The four modes for $\tilde{k}=3$ and $\tilde{d}=0.2$. Four neutral eigenvalues. 


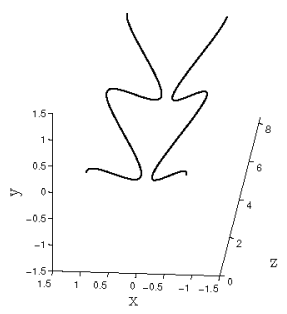

(a) Symmetric, growing

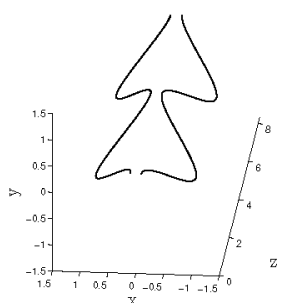

(b) Symmetric, decaying

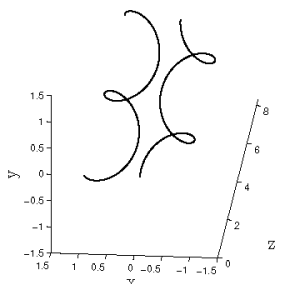

(c) Asymmetric, positive

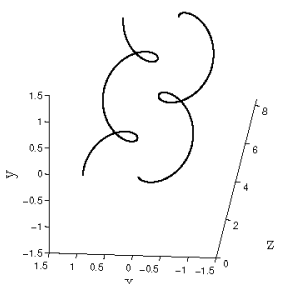

(d) Asymmetric, negative

Figure 4. The four modes for $\tilde{k}=1.5$ and $\tilde{d}=0.3$. Two neutral eigenvalues, one growing and one decaying.

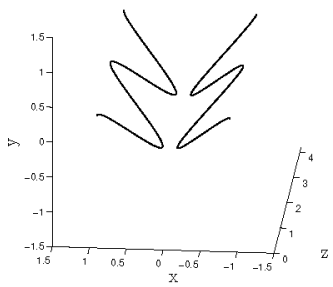

(a) Symmetric, growing

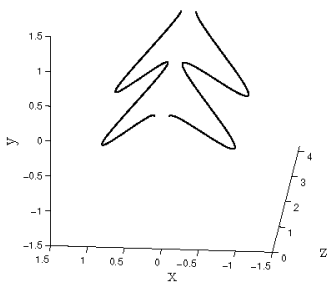

(b) Symmetric, decaying

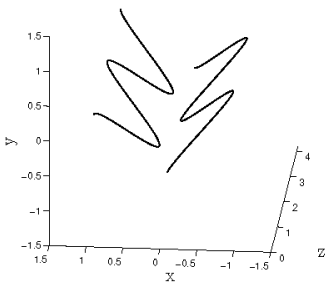

(c) Asymmetric, growing

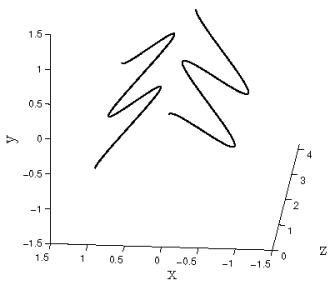

(d) Asymmetric, decaying

Figure 5. The four modes for $\tilde{k}=3$ and $\tilde{d}=0.4$. Two growing eigenvalues and two decaying.

parameter $q=\Gamma_{a} /\left(2 \pi W_{0}\right)$ that is what gives them the name.

$$
\begin{aligned}
& U(x, y)=-\left(1-e^{\frac{\left(x-X_{a}\right)^{2}+y^{2}}{a_{0}^{2}}}\right) \frac{y \Gamma_{a}}{2 \pi\left(\left(x-X_{a}\right)^{2}+y^{2}\right)}+\left(1-e^{\frac{\left(x+X_{a}\right)^{2}+y^{2}}{a_{0}^{2}}}\right) \frac{y \Gamma_{a}}{2 \pi\left(\left(x+X_{a}\right)^{2}+y^{2}\right)} \\
& V(x, y)=\left(1-e^{\frac{\left(x-X_{a}\right)^{2}+y^{2}}{a_{0}^{2}}}\right) \frac{\left(x-X_{a}\right) \Gamma_{a}}{2 \pi\left(\left(x-X_{a}\right)^{2}+y^{2}\right)}-\left(1-e^{\frac{\left(x+X_{a}\right)^{2}+y^{2}}{a_{0}^{2}}}\right) \frac{\left(x+X_{a}\right) \Gamma_{a}}{2 \pi\left(\left(x+X_{a}\right)^{2}+y^{2}\right)} \\
& W(x, y)=0 \\
& U(x, y)=-\left(1-e^{\frac{\left(x-X_{a}\right)^{2}+y^{2}}{a_{0}^{2}}}\right) \frac{y \Gamma_{a}}{2 \pi\left(\left(x-X_{a}\right)^{2}+y^{2}\right)}+\left(1-e^{\frac{\left(x+X_{a}\right)^{2}+y^{2}}{a_{0}^{2}}}\right) \frac{y \Gamma_{a}}{2 \pi\left(\left(x+X_{a}\right)^{2}+y^{2}\right)} \\
& V(x, y)=\left(1-e^{\frac{\left(x-X_{a}\right)^{2}+y^{2}}{a_{0}^{2}}}\right) \frac{\left(x-X_{a}\right) \Gamma_{a}}{2 \pi\left(\left(x-X_{a}\right)^{2}+y^{2}\right)}-\left(1-e^{\frac{\left(x+X_{a}\right)^{2}+y^{2}}{a_{0}^{2}}}\right) \frac{\left(x+X_{a}\right) \Gamma_{a}}{2 \pi\left(\left(x+X_{a}\right)^{2}+y^{2}\right)} \\
& W(x, y)=W_{0}\left(a+e^{\frac{\left(x-X_{a}\right)^{2}+y^{2}}{a_{0}^{2}}}\right)+W_{0}\left(a+e^{\frac{\left(x+X_{a}\right)^{2}+y^{2}}{a_{0}^{2}}}\right)
\end{aligned}
$$

Furthermore, these basic flows are not exact solutions of the Navier-Stokes equations, they are approximations. This will create some spurious results when calculating, but their importance can be minimized. One of the issues is that the descent speed of the vortices is not taken into account in these equations. This term is of order unity, so it should be added to the basic flow given here. Other important point is that they are inviscid solutions, and, as the real problem is viscous, the vortices will diffuse. If $R e$ is high enough, this will be negligible. Finally, these basic flows are analytic solutions for a single vortex, but not for the pair, so the closer that the two vortices are, the further that the result encountered will be from the actual one. This can be overcome by mean of a 2D DNS evolved some time steps from one of these basic flows until the shapes of the vortices remain unchanged in time. In that case, Re should be high for the vortices not to diffuse too much. This choice has been taken by many as González et al. ${ }^{-}$among others. 


\section{III.C.2. Boundary Conditions}

The boundary conditions for the variables $\hat{u}, \hat{v}$ and $\hat{w}$ are very easy to impose in the case that the two vortices are taken inside the domain and the boundaries are kept far away enough. In that case, Dirichlet conditions are imposed on all the boundaries. However, the symmetry of the problem allows to calculate the solution as the sum of symmetric and antisymmetric solutions of only one half. In that case, two different sets of conditions have to be imposed. Symmetry conditions mean Neumann boundary conditions, while antisymmetry mean Dirichlet boundary conditions.

Symmetric case: $\hat{v}$ and $\hat{w}$ are symmetric, while $\hat{u}$ is antisymmetric:

$$
\begin{aligned}
\hat{u}(x, y) & =-\hat{u}(-x, y) \\
\hat{v}(x, y) & =\hat{v}(-x, y) \\
\hat{w}(x, y) & =\hat{w}(-x, y)
\end{aligned}
$$

Antisymmetric case: $\hat{u}$ is symmetric, while $\hat{v}$ and $\hat{w}$ are antisymmetric:

$$
\begin{aligned}
\hat{u}(x, y) & =\hat{u}(-x, y) \\
\hat{v}(x, y) & =-\hat{v}(-x, y) \\
\hat{w}(x, y) & =-\hat{w}(-x, y)
\end{aligned}
$$

\section{III.C.3. Comparison Full boundary and Half Boundary}

First of all, a validation case for the boundary conditions is selected. It will be a case for low Re and $q$ of order unity, which lead some instabilities. The exact values of the problem are the following:

$$
R e=100, \quad q=0.475, \quad a=0.0, \quad X_{a}=2.0, \quad a_{0}=1.0, \quad \beta=0.418
$$

Convergence of the solution is shown in table 2 for the first two unstable eigenvalues. Good convergence is seen for both eigenvalues, but the symmetric boundary seems to have better behavior. Figure 6 highlights that the union of the symmetric and antisymmetric modes is the same result as the full solution. Similarities can be found with figure $4 \mathrm{~b}$ of Hein and Theofilis. These spectra have been calculated with high order finite differences of order 8. The Krilov space was only of 100 points, and that is the reason because the number of eigenvalues is not very big and the continuous part of it is not very well converged, although it is not needed either.

The modes are complex and therefore, as the grid is not the same for the case of full domain and the case of half domain, the eigenvectors are moved one phase and then they do not look the same for half and full domain. However, the reconstruction in space and time will give the same Figures 7 and 8 represent the value of the imaginary part of the modes, only for the full domain simulations. There, the kind of boundary condition in the center is appreciated, where it can be seen that all the variables, but $\hat{u}$ follow the symmetry or antisymmetry of the mode, while $\hat{u}$ does the opposite.

\section{III.C.4. Effect of adding the descent speed}

The results presented before are calculated without adding the descent speed of the vortices to the basic flow and for an analytic and imposed base flow. In this paragraph it is shown how the descent speed, $V_{d}=q / 2 X_{a}=0.119$, even if small, displaces the spectrum. For that purpose, figure 9 is shown, where convergence have been analyzed again, as well as the symmetric and antisymmetric meshes are used to show whether the eigenvalues are symmetric or antisymmetric. The shape of the first two modes (not shown here) is very similar to those without descent speed added (again symmetric and antisymmetric respectively), and therefore these two modes correspond to the ones with descent speed equal zero.

\section{Conclusion}

Both methods have been proved to be able to recover good results in the analysis of two counter rotating vortices. Point vortices were already analyzed long time ago, but this methodology offers a fast and reliable tool to compare with others in the inviscid limit. The viscous BiGlobal stability analysis is a established 
Table 2. Table of convergence for the two more unstable modes.

\begin{tabular}{lrrcccc} 
Mesh & $N_{x}$ & $N_{y}$ & Mode $1 \omega_{i}$ & Mode $1 \omega_{r}$ & Mode $2 \omega_{i}$ & Mode $2 \omega_{r}$ \\
\hline Symmetric & 150 & 300 & 0.103125944794 & 0.042288780713 & - & - \\
Antisymmetric & 150 & 300 & - & - & 0.083025955469 & 0.057975378840 \\
Full & 200 & 200 & 0.103125948637 & 0.042288786637 & 0.083026013284 & 0.057969180155 \\
Symmetric & 100 & 200 & 0.103125945214 & 0.042288785006 & - & - \\
Antisymmetric & 100 & 200 & - & - & 0.083026064183 & 0.057975206122 \\
Full & 150 & 150 & 0.103125943203 & 0.042288810039 & 0.083026004911 & 0.057969173492 \\
Symmetric & 75 & 150 & 0.103125957635 & 0.042288817958 & - & - \\
Antisymmetric & 75 & 150 & - & - & 0.083025996071 & 0.057963434941 \\
Full & 100 & 100 & 0.103126030491 & 0.042289023553 & 0.083026144726 & 0.057969326685 \\
Symmetric & 50 & 100 & 0.103126311524 & 0.042289742338 & - & - \\
Antisymmetric & 50 & 100 & - & - & 0.083026837579 & 0.057975629592 \\
Full & 50 & 50 & 0.103142154419 & 0.042328587689 & 0.083062987914 & 0.058011763106
\end{tabular}

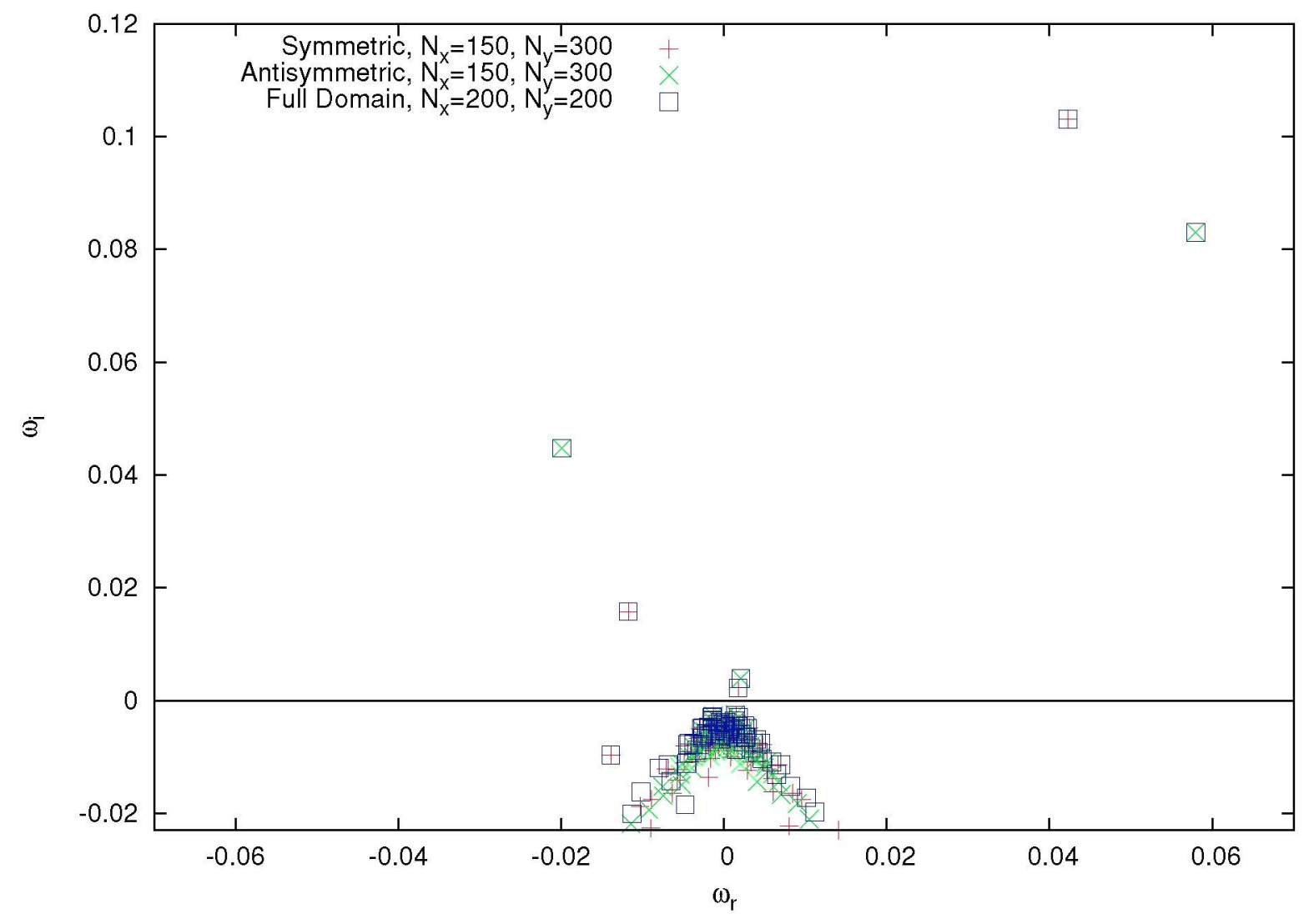

Figure 6. Spectrum obtained for both domains. 


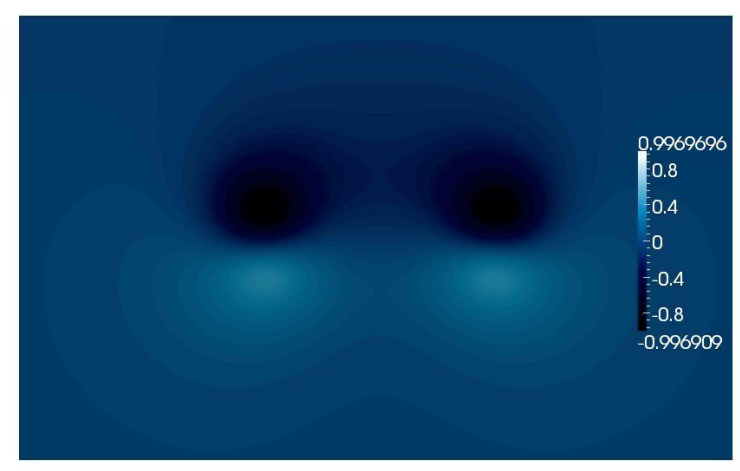

(a) Pressure.

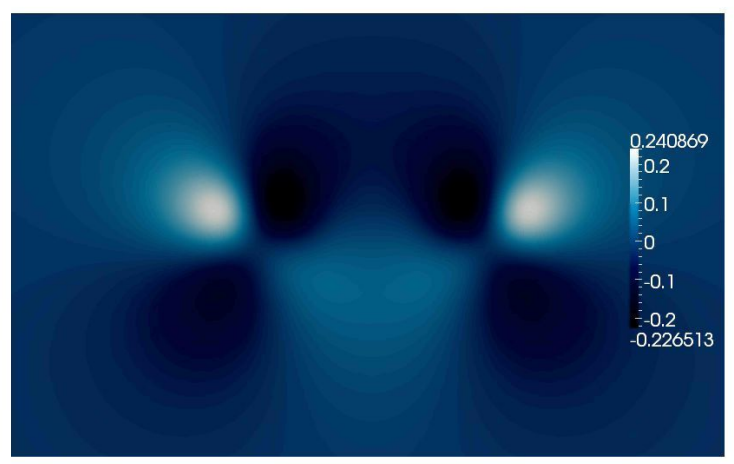

(c) Velocity in y direction.

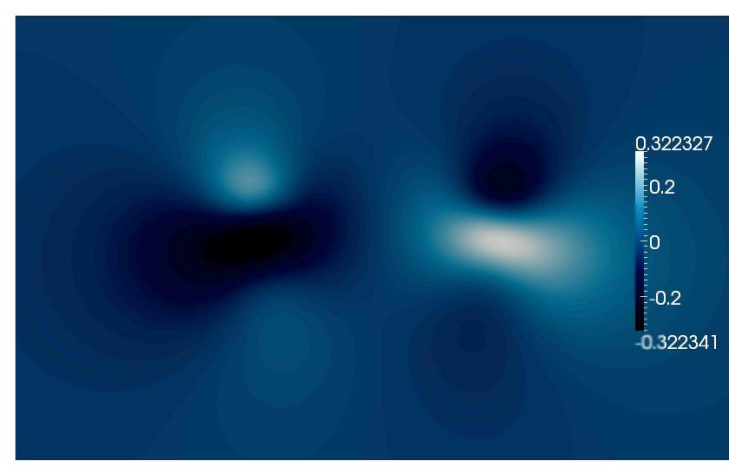

(b) Velocity in $\mathrm{x}$ direction.

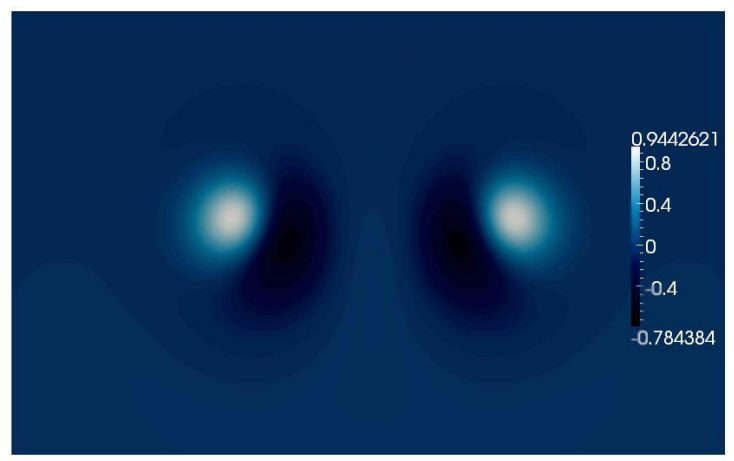

(d) Velocity in $z$ direction.

Figure 7. Imaginary part of the four components of the first symmetric mode for the values specified above.

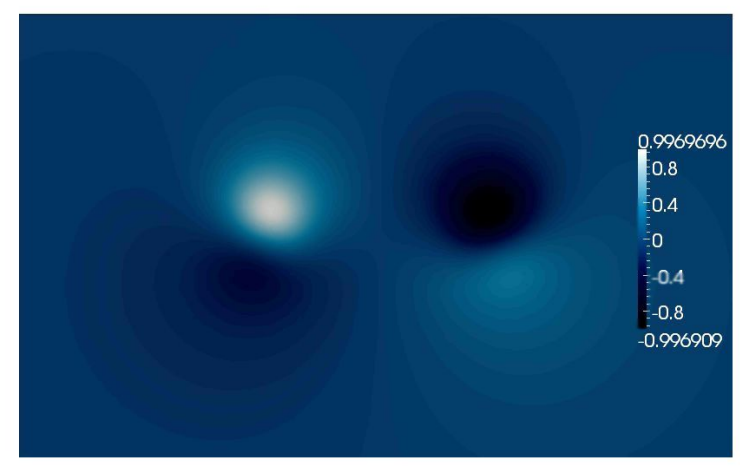

(a) Pressure.

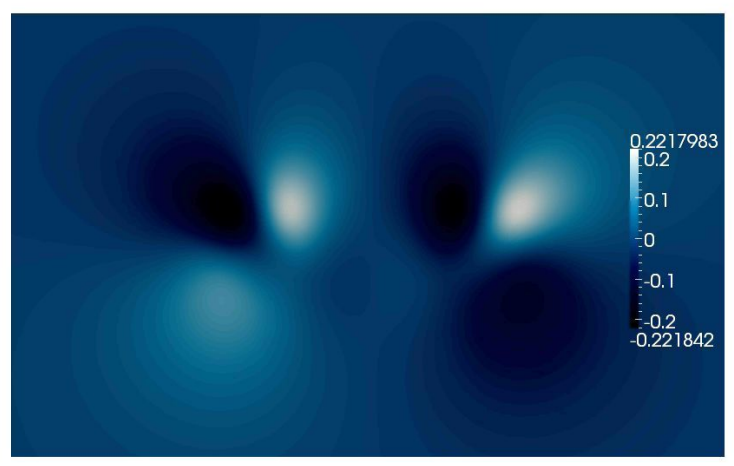

(c) Velocity in y direction.

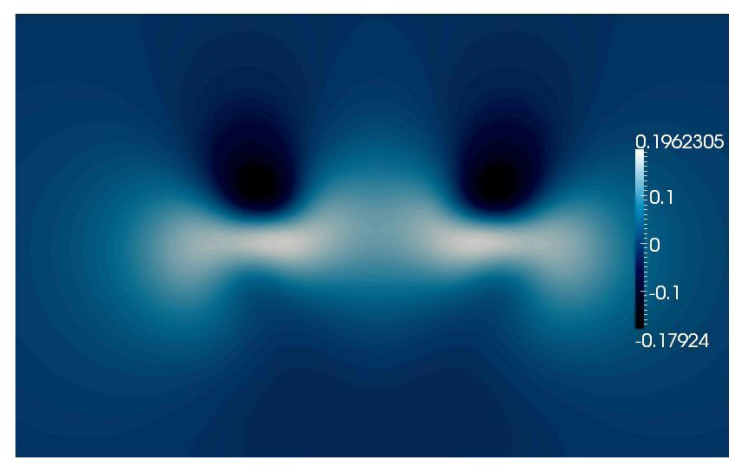

(b) Velocity in $\mathrm{x}$ direction.

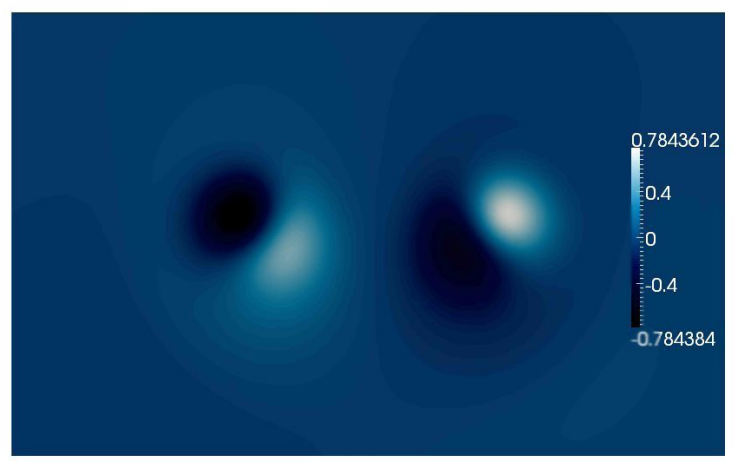

(d) Velocity in $z$ direction.

Figure 8. Imaginary part of the four components of the first antisymmetric mode for the values specified above. 


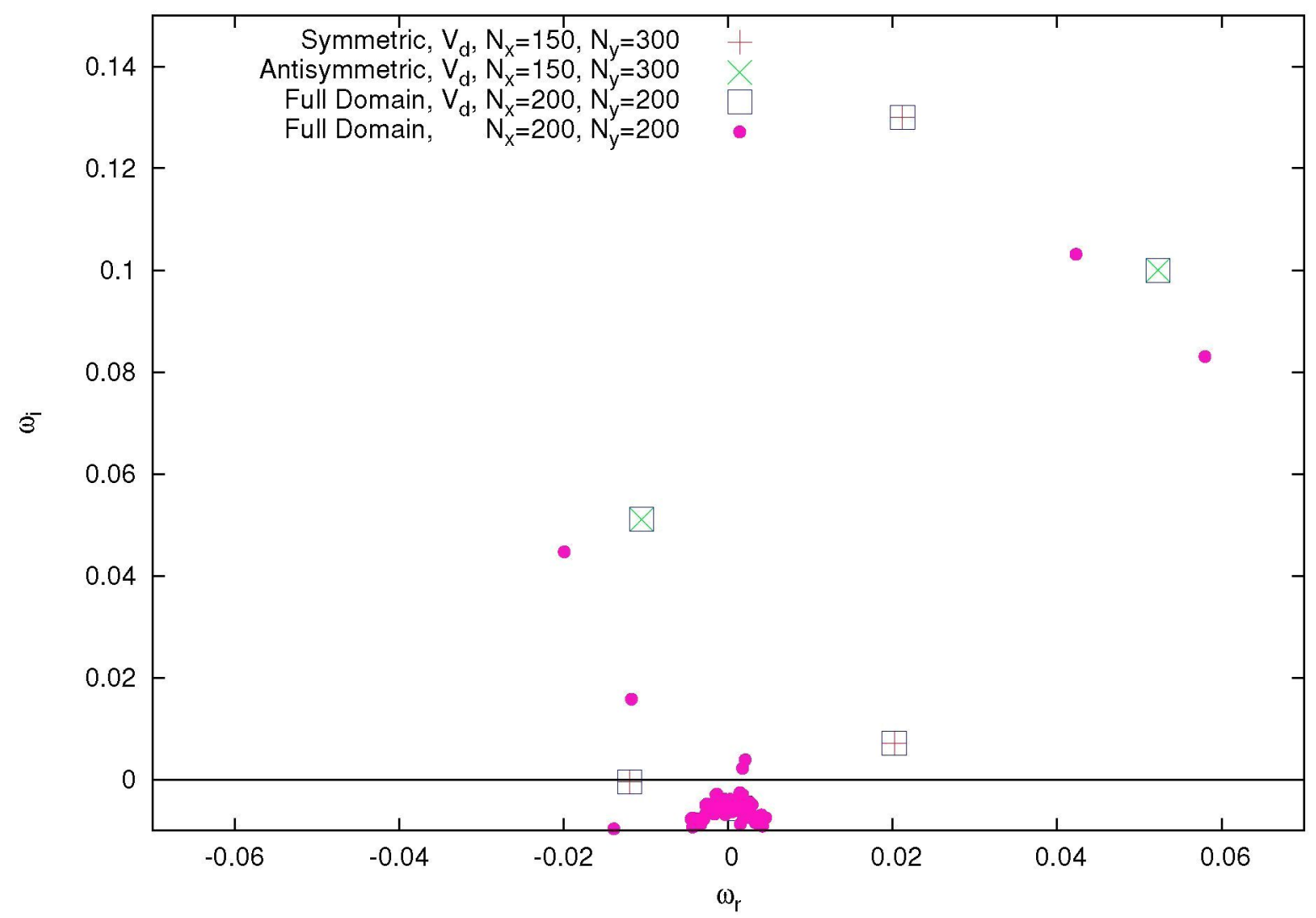

Figure 9. Spectrum to show the effect of the descent speed, in this case, $V_{d}=0.11875$ 
methodology, which, also in this case, have demonstrated that the use of this new upgrade can reproduce or even improve previous results with very little computational resources.

The advantages of the point vortex stability methods are the simplicity, which means easy to compute, and the small number of parameters. By contrast, due to that facts, the real problems cannot be modeled accurately.

The BiGlobal method, on the other hand, offers a wide range of parameters to analyze, and different options when modeling the equations. These are both its advantages and disadvantages, because the number of parameters, among them the viscosity, allows the possibility to model the problem with more accuracy, but if not done properly, might not lead to interesting conclusions. In that line, this work has analyzed the effect of some of the parameters, but much more can be done in that area.

\section{Acknowledgments}

Support of the Secretaría de Estado de Investigación, Desarrollo e Innovación of the Spanish Ministry of Economy and Competitiveness through Grant MICINN-TRA2009-13648: "Metodologias computacionales para la predicción de inestabilidades globales hidrodinámicas y aeroacústicas de flujos complejo" is gratefully acknowledged.

\section{References}

${ }^{1}$ Crow, S. C., "Stability Theory for a Pair of Trailing Vortices," AIAA J., Vol. 8, 1970, pp. 2172-2179.

${ }^{2}$ Crouch, J. D., "Instability and Transient Growth for Two Trailing-Vortex Pairs," J. Fluid Mech., Vol. 350, 1997, pp. 311330.

${ }^{3}$ Kármán, T. V., "Über den mechanismus des widerstandes, den ein bewegter körper in einer flüssigkeit erfährt," Nachr. Ges. Wissenschaft. Göttingen, 1911, pp. 509-517.

${ }^{4}$ Kármán, T. V., "Über den mechanismus des widerstandes, den ein bewegter körper in einer flüssigkeit erfährt," $N a c h r$. Ges. Wissenschaft. Göttingen, 1912, pp. 547-556.

${ }^{5}$ Jiménez, J., "Stability of a pair of co-rotating vortices," Phys. Fluids, Vol. 18 (11), 1970, pp. 1580-1581.

${ }^{6}$ Fabre, D., Cossu, C., and Jacquin, L., "Spatio-temporal development of the long and short-wave vortex-pair instabilities," Phys. Fluids, Vol. 12, No. 5, MAY 2000, pp. 1247-1250.

${ }^{7}$ Hein, S. and Theofilis, V., "On instability characteristics of isolated vortices and models of trailing-vortex systems," Comp. \& Fluids, Vol. 33, No. 5-6, JUN-JUL 2004, pp. 741-753, Conference on Applied Mathematics for Industrial Flow Problems, LISBON, PORTUGAL, APR 17-JUL 20, 2002.

${ }^{8}$ Rodríguez, D. and Theofilis, V., "Massively Parallel Solution of the BiGlobal Eigenvalue Problem Using Dense Linear Algebra," AIAA J., Vol. 47, No. 10, OCT 2009, pp. 2449-2459, AIAA 46th Aerospace Sciences Meeting and Exhibit, Reno, NV, JAN 07-10, 2008.

${ }^{9}$ Broadhurst, M., Theofilis, V., and Sherwin, S., "Spectral element stability analysis of vortical flows," Sixth IUTAM Symposium on Laminar-Turbulent Transition, edited by Govindarajan, R, Vol. 78 of Fluid Mechanics and its Applications, Int Union Theoret \& Appl Mech, SPRINGER, PO BOX 17, 3300 AA DORDRECHT, NETHERLANDS, 2006, pp. 153-158, 6th IUTAM Symposium on Laminar-Turbulent Transition, Bangalore, INDIA, DEC 13-17, 2004.

${ }^{10}$ Gonzalez, L. M., Gomez-Blanco, R., and Theofilis, V., "Eigenmodes of a Counter-Rotating Vortex Dipole," AIAA J., Vol. 46, No. 11, NOV 2008, pp. 2796-2805, AIAA 37th Fluid Dynamics Conference, Miami, FL, JUN 25-28, 2007.

${ }^{11}$ Paredes, P., Hermanns, M., Clainche, S. L., and Theofilis, V., "Order $10^{4}$ speedup in global linear instability analysis using matrix formation," Comput. Methods Appl. Mech. Engrg., Vol. 253, 2013, pp. 287-304.

${ }^{12}$ Rennich, S. C. and Lele, S. K., "Method for Accelerating the Destruction of Aircraft Wake Vortices," Journal of Aircraft, Vol. 36, No. 2, 1999, pp. 398-404.

${ }^{13}$ Widnall, S. E., Bliss, D. B., and Zalay, A., Aircraft Wake Turbulence and its Detection, Plenum, New York, 1971, p. 305.

${ }^{14}$ Theofilis, V., "Advances in Global Linear Instability Analysis of Nonparallel and Three-Dimensional Flows," Prog. in Aero. Sciences, Vol. 39 (4), 2003, pp. 249-315.

${ }^{15}$ Theofilis, V., "Global linear instability," Annu. Rev. Fluid Mech., Vol. 43, 2011, pp. 319-352.

${ }^{16}$ Butler, K. and Farrell, B. F., "Three-Dimensional Optimal Perturbations in Viscous Shear Flow," Physics of Fluids, Vol. 4 (8), 1992.

${ }^{17}$ Mack, L. M., "Boundary-Layer Stability Theory" AGARD Report No. ro9. Special Course on Stability and Transition of Laminar Flow, 1984, pp. 3-1-3-81. 